\title{
RESEÑA: DONNE E SCIENZA DALL'ESCLUSIONE AL PROTAGONISMO CONSAPEVOLE. ANTONELLA CAGNOLATI E SANDRA ROSSETTI ${ }^{1}$
}

Fiorenza Taricone ${ }^{2}$

\begin{abstract}
Antonella Cagnolati e Sandra Rossetti (ed.), Donne e scienza dall'esclusione al protagonismo consapevole. Roma: Aracne, 2016. ISBN: 978-88-548-8764-0.

Il libro curato da Antonella Cagnolati e Sandra Rossetti, pubblicato nella Collana Donne nella storia. Segni, tracce, percorsi, si occupa di un tema centrale da anni ormai negli studi di genere italiani ed europei. Peraltro, l'approccio delle giovani generazioni verso le discipline esatte, è ancora in taluni campi a svantaggio delle ragazze, penalizzandole poi a livello lavorativo, perché l'offerta di lavoro è decisamente maggiore nei settori scientifici, piuttosto che umanistici; il Programma europeo STEM Disciplines, (Tecnologia, Ingegneria, Matematica), è stato ideato proprio per stimolare le giovani a considerare seriamente la possibilità per il loro futuro di scegliere corsi di studio nelle discipline ancora poco visitate; uno zoccolo duro rappresentato dalla Fisica, dall'Ingegneria aero spaziale, dalle nano tecnologie, per fare degli esempi, rispetto ad altre nelle quali il sorpasso femminile, nelle iscrizioni e anche nei risultati è già avvenuto, come Medicina.
\end{abstract}

L'esclusione è antica, e a mio giudizio doppia: se l'ingresso nei circuiti ufficiali del sapere era proibito, tranne poche eccezioni come Ipazia, le scuole pitagoriche, le monache colte, le figlie, sorelle o mogli di scienziati, a causa della ontologica deficienza del sesso femminile, l'accreditamento delle donne in alcuni settori scientifici li svalutava per definizione.

\footnotetext{
${ }^{1}$ Data di ricezione: 08/10/2019.

Data di accettazione: 10/10/2016.

${ }^{2}$ Associata di Storia delle Dottrine Politiche e Pensiero Politico e Questione Femminile, Università degli studi di Cassino e del Lazio Meridionale, Italia; $₫$ f.taricone@unicas.it.
} 
Superfluo forse ricordare le competenze sulle proprietà curative delle erbe, un vero reato per le streghe nel Cinque-Seicento, un settore economicamente trainante oggi, o l'arte di far nascere i figli, praticata da dilettanti dell'ostetricia, rispetto alla professionalità dei medici curanti e laureati. Nell'Introduzione, Antonella Cagnolati e Sandra Rossetti definiscono non a caso la scienza un "mitico universo autoreferenziale, custodito da feroci guardiani della soglia".

Il libro, articolato in saggi a firma di donne e uomini, di cui uno in inglese a firma di Tamar Groves, Estrella Montes e Francisco J. Rodriguez, parte cronologicamente con l'Ottocento per poi proseguire con la contemporaneità. La battuta d'inizio è riservata a un tema scottante per le donne, l'isteria, collegata alle cure mesmeriche, Donne magnetiche. Il femminile nel mesmerismo. Il saggio di Luigi Traetta prende le mosse dagli esperimenti iniziali di Mesmer che nel 1773 iniziò un trattamento con Franziska Oesterling, fidanzata di Franz de Paula von Bosh, figlio della vedova Maria Anna von Eulenschenk, che aveva sposato lo stesso Mesmer. Il successo convinse quest'ultimo che era la calamita l'unico mezzo a possedere le presunte proprietà terapeutiche idonee a ricreare nel corpo umano un qualcosa di simile alle maree. La sostanza magnetica si propagava come un fluido elettrico per mezzo di corpi intermediari come vari metalli, carta, pane, lana, acqua, legno e soprattutto cani ed esseri umani. Nel 1777, Mesmer e sua moglie avevano trasformato la loro abitazione in clinica specializzata per la cura di convulsioni, emiplegie, disturbi ginecologici e soprattutto oftalmie. La loro vita professionale non fu facile, dovendo fare i conti con il pregiudizio che i soggetti da curare fossero principalmente donne, perché come disse a Freud un vecchio medico, hysteron voleva dire utero e quindi non poteva riguardare gli uomini. Charcot invece si era convinto che l'origine dell'isteria fosse essenzialmente traumatica e ideogena. L'Autore descriveva la sessualità di un paziente interamente condizionata dall'attrazione per i chiodi presenti sulle scarpe femminili. Nel periodo in cui vedeva meno donne era meno eccitato, al contrario, durante le vacanze, in loro compagnia, il malessere aumentava. I chiodi erano quindi l'idea che aveva originato lo stato di malessere dall'età di sei anni; la via per curare l'isteria era quindi nell'identificazione ed eliminazione dell'idea traumatica. 
Il secondo saggio ottocentesco è quello di Bruna Bianchi, Scienza e spiritualità nel pensiero di Anna Bonus Kingsford; femminista e filosofa, interessata allo spiritualismo e all'ermetismo, tra le prime donne inglesi a conseguire la laurea in Medicina, (è visibile anche una sua foto nel volume); criticò per tutta la sua vita la scienza tradizionale, mettendo in relazione la violenza sugli animali, il dominio sulle donne, l'etica della professione medica, la dietologia e il consumo, personalmente convinta com'era di un legame inscindibile fra tutti gli esseri viventi. Il suo primo impatto con la vivisezione era narrato da lei stessa: mentre stava studiando al Museo di Storia Naturale udì i guaiti "strazianti" di un cane torturato.

Il saggio di Cristina Romano è invece strettamente contemporaneo e riguarda l'affascinante figura di Rita Charon, medico internista e docente di Clinica Medica alla Columbia University di New York, alla quale nel 2011 l'Ala Dea Morani Renaissance Woman Award, Fondazione statunitense per la storia delle donne nella medicina, ha riconosciuto il premio. Nata nel 1949, a Providence, nel Rhode Island, padre medico generico, inizia come insegnante di biologia e padagogia, affiancando il vlontariato come attivista pacifista. Poi, nel 1974, data evidentemente cui non è estranea la rivoluzione femminista, decide di laurearsi anche in medicina. La sua carriera, scrive l'Autrice, si sviluppa seguendo una direzione precisa, quella della medicina narrativa che si propone di considerare rilevante ai fini della diagnosi e della cura, la narrazione della malattia effettuata dal/la paziente; a Rita Charon appartiene anche la definizione stessa, cioè medicina narrativa, da cui è nato l'acronimo in lingua inglese NBM, ovvero Narrative Based Medicine che rimanda all'altro acronimo tadizionale EBM, Evidence Based Medicine; quest'ultima privilegia il conosciuto/conoscibile/ l'universale e il corpo, la seconda il non conosciuto/non conoscibile, il particolare, il sé.

Fulvia Signani titola invece il suo intervento Policy di genere per la salute, partendo dalla Medicina di genere "che non rappresenta una disciplina medica a parte, ma un nuovo modo d'intendere la prevenzione, diagnosi e cura delle malattie, in ottica di appropriatezza ed equità”. Gli stereotipi negativi ancora predominanti sul genere influenzano molto la pratica clinica. Le policies si rivelano quindi utili perché non fanno riferimento solo a leggi, ma anche a linee guida e regolamenti. Attraverso una serie di 
grafici piramidali, l'Autrice evidenza i contrasto agli stereotipi, e l'inclusione del genere attraverso tappe quali l'accettazione dell'incompetenza anche temporanea, l'apertura al nuovo, l'accreditamento di ricerche e pubblicazioni, e finanziamenti di ricerche solo se trattano il genere, favorendo pratiche di salute "gender oriented".

Paola Conti si occupa di Engendering Health, inteso, come scrive, nel senso di un processo dinamico dell'intendere e agire la salute in ottica di genere. Per i professionisti della salute comprendere le interconnessioni tra sesso, genere e salute significa poter sviluppare nuove competenze strategiche relative all'efficacia e alla dignità di genere. Quindi, come nel saggio precedente, il richiamo è una positiva curiosità di nuovi saperi, che deve fare i conti con il potere, "dimensione chiave" nella costruzione dei saperi stessi. Anche in questo saggio, grafici completano lo scritto, ad esempio un modello concettuale di transdisciplinarietà; un approccio di questo tipo tende a sottolineare "l'importanza della riflessività, strettamente connessa alla consapevolezza che il potere e il controllo sull'oggetto di studio è influenzato anche dalla posizione sociale dei ricercatori impegnati nel processo di co-produzione di conoscenza".

Amelia Ceci riporta in questa sede la relazione al V Seminario di Studi di Genere, Università di Ferrara, 2015, dedicato a Donne e scienza dall'esclusione al protagonismo consapevole, che è anche il titolo del volume recensito. Nel 2009 l'Autrice aveva incontrato durante una visita di studio in Svezia Karin Schenk-Gustafsson, che parlava della necessità di superare il "bikini view" rispetto alla salute della donna; la tendenza cioè a considerare solamente seno e organi riproduttivi come specificità della salute femminile. Pensare solo in termini riproduttivi portava a trascurare il fatto che ci fossero altre patologie di cui le donne soffrivano, ad esempio le patologie reumatiche, cardiovascolari, la sclerosi multipla, le disfunzioni tiroidee, l'ipertensione polmonare. Nel settembre del 2010, la stessa Autrice aveva partecipato alla Summer School europea Eugim di Berlino, dove era emerso chiaramente che la disparità di genere insita nella civiltà soprattutto occidentale aveva influenzato la scienza, la medicina e l'ingegneria, riducendo il beneficio che potevano apportare alla società. Nella stessa Summer School, Ineke Klinge e Flavia Franconi avevano mostrato chiaramente come la ricerca bio medica fosse stata per lungo tempo incentrata su un soggetto ideale, maschio, bianco, occidentale, 
di $70 \mathrm{~kg}$. trascurando le caratteristiche di genere e di sesso. Nel saggio è riportato sinteticamente il risultato di un lavoro di ricerca dell'Autrice, del 2014, che ha voluto dare un contributo a superare "il modello a taglia unica".

Beatrice Zucchi e Roberto Manfredini invece con il saggio Genere e ritmi ci introducono nell'interessante scienza dei ritmi biologici, la cronobiologia. "Una disciplina delle scienze bio-mediche dedicata allo studio della variabilità delle funzioni di un organismo in rapporto al tempo, organizzate nei cosiddetti ritmi biologici”. Se la prima osservazione al riguardo risale al 300 a.C., quando un naturalista scienziato al seguito di Alessandro Magno riportò negli appunti come le foglie del tamarindo presentassero un movimento ciclico corrispondente all'alternanza luce-buio, oggi le funzioni degli esseri viventi oscillano secondo un ritmo endogeno, con un massimo e un minimo. A seconda della lunghezza i ritmi biologici sono classificati in tre tipi principali: ritmi circadiani, con un periodo di 24 ore; ultraradiani, periodo inferiore alle 24 ore; infradiani, superiore alle 24 ore. Il primo orologio circadiano, posizionato nell'ipotalamo, è regolato dall'alternanza luce-buio. In base a questi ritmi, gli Autori analizzano i cronotipi, cioè la propensione degli esseri umani a essere maggiormente attivi in un particolare periodo della giornata; i soggetti quindi possono essere divisi in mattutini o allodole, e serotini, o gufi. Il saggio analizza quindi in maniera molto interessante in base al cronotipo e alle attività del quotidiano, le differenze di genere.

Sandra Rossetti, una delle due curatrici, dedica il suo saggio a La camicia con le pin-up. Eco femminismo e scienza delle donne. Nel 2014 erano apparsi su alcune testate giornalistiche italiane articoli dedicati a Matt Taylor, fisco inglese dell'Agenzia Spaziale europea, capo del progetto Rosetta, una sonda spaziale destinata allo studio delle comete. Lo scienziato aveva attirato l'attenzione sulle sue camicie sgargianti, con immagini di pin-up senza veli; era stato attaccato per questo, accusato di misoginia, e Taylor aveva reagito scoppiando in lacrime e chiedendo scusa; per l'Autrice era una chiara dimostrazione di come gli stereotipi fossero vivi e vegeti anche in uomini con un background di elevato livello e anche "un'ennesima prova di come questo fenomeno riguardi lo stesso vissuto degli scienziati, i quali, per la rivendicata obiettività ed oggettività del loro stile conoscitivo, dovrebbero essere invece totalmente immuni”. A 
poco quindi erano valsi i programmi europei come Gendered innovations del 2011, che proponeva esempi e modelli per tenere nella dovuta considerazione la differenza sessuale nei più svariati ambiti della ricerca: medicina, biologia, clima, inquinamento, tecnologie.

Tamar Groves, Estrella Montes e Francisco J. Rodriguez iniziano con il sottolineare le difficoltà comuni che le donne incontrano in tutti i paesi europei nelle carriere, soprattutto in tre momenti: scelta degli studi, i primi passi nelle carriere scientifiche e il loro consolidamento. In modo particolare, hanno incentrato la ricerca su donne e uomini che avevano ricevuto borse Fulbright dal 1959 al 1974, quindi evidentemente sotto il regime franchista. Nel periodo analizzato, erano presenti molti più uomini che donne nei grantees, per di più con una spiccata presenza femminile nelle discipline umanistiche, in rapporto di 75 a $10 \%$. Tutte le persone coinvolte erano del parere che l'esperienza dei grants all'estero fosse stata una chiave importante per lo sviluppo professionale, ma anche che il ritorno in Spagna fosse stato traumatico.

Infine, il saggio di Cristiana Fioravanti su Il bilancio di genere. Strumento chiave di monitoraggio dei percorsi femminili in ambito universitario. Inizia con un più che giusto riconoscimento all'Europa per aver supportato la dimensione di genere all'interno delle Università italiane. Cionostante, le sperequazioni sono ancora molte e ben note, a partire dal fatto che le studentesse superano da tempo gli immatricolati, tranne che nelle scienze dure, si laureano prima e meglio, ma le loro competenze si disperdono dopo la laurea e che nelle Università nelle posizioni apicali le donne sono poche rispetto al totale: molte ricercatrici, poche ordinarie. Stessa rarefazione negli organi di governo dell'Ateneo. A partire dal 2011 in relazione a questo, l'Università di Ferrara ha inziato un percorso di rendicontazione annuale delle dinamiche di genere fra le proprie componenti: studenti, docenti e ricercatori, assegnisti, dottorando, personale tecnicoamministrativo. Il bilancio di genere ha costituito in questo un punto di partenza imprescindibile. 